ESTUDIOS

\title{
DISERTACIONES
}

Grupos minoritarios y estigmatizados: diversidad funcional, religiosa, étnica, afectivo-sexual o de identidad de género en la comunicación

ISSN: $1856-9536$

Doi: https://doi.org/10.12804/revistas.urosario.edu.co/disertaciones/a.10388

Volumen 15, Número 1 / Enero-junio 2022

Versión PDF para imprimir desde

http://revistas.urosario.edu.co/index.php/disertaciones

Para citar este artículo: Cuenca Orellana, N., \& Martínez Pérez, N. (2022). Adolescencia y homosexualidad en las series de ficción: análisis narrativo de Esta mierda me supera y A Million Little Things. Anuario Electrónico de Estudios en Comunicación Social "Disertaciones", 15(1), 1-14. https://doi.org/10.12804/ revistas.urosario.edu.co/disertaciones/a.10388

\section{ADOLESCENCIA Y HOMOSEXUALIDAD EN LAS SERIES DE FICCIÓN: ANÁLISIS NARRATIVO DE ESTA MIERDA ME SUPERA Y A MILLION LITTLE THINGS}

Adolescence and Homosexuality in Fiction Series: Narrative Analysis of I Am Not Okay with This and A Million Little Things

Adolescência e homossexualidade em séries de ficção: análise narrativa de I Am Not Okay with This e A Million Little Things

Nerea Cuenca Orellana, Universidad Rey Juan Carlos (España)

nerea.cuenca@urjc.es

Natalia Martínez Pérez, Universidad Carlos III de Madrid (España)

nmperez@hum.uc3m.es

Recibido: 15 de marzo del 2021

Aprobado: 26 de agosto del 2021

Fecha de prepublicación: 27 de enero del 2022

\section{RESUMEN}

En las últimas dos décadas, las nuevas vías en las cuales se proyectan las series de ficción, tales como Internet y las plataformas Video-on-demand (vod), han abierto el abanico de las representaciones de género. La inclusión de personajes lgbtq+ tanto protagónicos como secundarios en la narrativa serial parece estar superada. El presente 


\title{
DISERTACIONES
}

Grupos minoritarios y estigmatizados: diversidad funcional, religiosa, étnica, afectivo-sexual o de identidad de género en la comunicación

ISSN: $1856-9536$

Doi: https://doi.org/10.12804/revistas.urosario.edu.co/disertaciones/a.10388

Volumen 15, Número 1 / Enero-junio 2022

Versión PDF para imprimir desde

http://revistas.urosario.edu.co/index.php/disertaciones

trabajo ofrece un análisis de dos series de televisión recientes con personajes adolescentes homosexuales: A Million Little Things y Esta mierda me supera. Con la finalidad de observar el tratamiento que se da a estos personajes, se realizó un análisis de contenido narrativo desde el punto de vista de la dimensión psicológica, de acuerdo con el modelo metodológico de Carlos Grossocordón. Los resultados demuestran ciertas diferencias de género en las representaciones de adolescentes lesbianas y gays, pero, en definitiva, ofrecen nuevos y positivos retratos de la homosexualidad alejados de estereotipos, que dan más visibilidad al colectivo lgbtq+ y brindan una mayor aceptación por parte de la audiencia.

Palabras clave: género; adolescentes; homosexualidad; Esta mierda me supera; A Million Little Things; series de televisión.

\begin{abstract}
In the last two decades, new avenues in which to screen fiction series, such as the Internet and Video-on-demand platforms, have broadened the range of genre representations. The inclusion of both secondary and leading lgbtq+characters in serial narratives seems successful. The present work analyzes two recent television series with homosexual adolescent characters: A Million Little Things and I Am Not Okay with This. To evaluate how these characters are portrayed, narrative content analysis is carried out from the psychological perspective, following Carlos Grossocordón's methodological model. The results reveal certain gender differences in the representations of adolescent lesbians and gays; however, they provide new and positive portraits of homosexuality, away from stereotypes, which offer greater visibility to the Igbtq+ community and are more widely accepted by the audience.
\end{abstract}

Keywords: Gender; adolescents; homosexuality; I Am Not Okay with This; A Million Little Things; tv series.

\section{RESUMO}

Nas últimas duas décadas, os novos caminhos para a projeção de séries de ficção, como a internet e as plataformas de vídeo On Demand, abriram espaço para as representações de gênero. A inclusão de personagens lgbtq+ secundários e principais na narrativa serial está desatualizada. O presente trabalho oferece uma análise de duas séries de televisão recentes com personagens adolescentes homossexuais: A Million Little Things e I Am Not Okay with This. Para observar o tratamento dispensado a esses personagens, é realizada uma análise de conteúdo narrativa do ponto de vista da dimensão psicológica, de acordo com o modelo metodológico de Carlos Grossocordón. Os resultados mostram certas diferenças de gênero nas representações de adolescentes lésbicas e gays, mas, em suma, oferecem novos e positivos retratos da homossexualidade longe dos estereótipos, que dão maior visibilidade à comunidade lgbtq+e proporcionam maior aceitação pelo público.

Palavras-chave: gênero; adolescentes; homossexualidade; Essa merda sobre mim; Um milhão de pequenas coisas; série de televisão. 


\section{DISERTACIONES}

ESTUDIOS

\section{Introducción}

El nuevo milenio ha traído consigo cambios en la percepción social de la homosexualidad. Los contenidos audiovisuales, entendidos como herramienta de socialización y medios de transmisión de cambios en la sociedad del momento, se han ido adaptando a las demandas de un público cada vez más diversificado y exigente. Las historias contemporáneas se centran más en aspectos normativos que descriptivos, es decir, las narraciones cuentan cómo el mundo debería ser en relación a las normas culturales, en lugar de como es en realidad (Veissière, 2018). Conocer las expectativas y preferencias de la adolescencia así como descubrir la forma en la que incorporan el consumo de ficción en su día a día, facilita el diseño de producciones audiovisuales específicas con los que entretenerse y, a su vez, les proporcionan elementos educativos o formas de actuar y relacionarse en su cotidianeidad (Fedele \& García-Muñoz, 2010). Esto también se traslada al público adulto, pues tener acceso a representaciones homosexuales adolescentes puede ofrecer recursos a la hora de tratar el tema con sus hijos o hijas, al igual que abordar las complicaciones que puedan surgir en las relaciones paterno-filiales durante este periodo.

La llegada de canales de televisión centrados en la emisión de series de ficción, sin duda, ha sido uno de los avances fundamentales para los espectadores de todas las edades, y más aún, para el público adolescente que necesita sentirse representado en estos contenidos. Buscar y desarrollar su propia identidad es clave en el desarrollo humano, donde el concepto social de género juega un gran papel. La sociedad occidental cada vez consume más series de ficción y se considera que estas presentan algunas funciones sociales fundamentales (Von Feilitzen, 2008). Cada espectador/a busca identificarse en la historia con los personajes y con sus emociones, del mismo modo que reinterpreta la narración según sus propias experiencias. Al fin y al cabo, mediante la observación, identificación e imitación, los seres humanos perpetuamos actitudes o acciones (Pacheco Mejía, 2007).

Puede señalarse que lo realmente importante de una representación no es la idea general, sino que su valor reside en el contenido material y semántico que se le da a una determinada imagen o arquetipo narrativo (Durand, 2004). En tal sentido, desde el punto de vista narrativo el presente trabajo aborda cómo se representa en la actualidad la homosexualidad adolescente, en este caso, a partir del análisis en dos series de ficción estadounidenses: A Million Little Things (ABC, 2018) y Esta mierda me supera (I Am Not Okay with This, Netflix, 2020). La primera, creada por D.J. Nash, cuenta cómo un grupo de amigos en Boston, que se reúnen después de un tiempo, se dan cuenta de que deben dejar las vidas en las que se sienten atrapados después de que uno de ellos muriera inesperadamente, esto es, se suicidara. El hijo del fallecido, Danny Dixon (Chance Hurstfield), es un chico gay de doce años que vive su cotidianeidad y da visibilidad a este colectivo en una serie destinada a un público adulto. La segunda, clasificada como serie para adolescentes o Teen Series, está basada en la novela gráfica homónima y ha sido adaptada a televisión por Jonathan Entwistle y Christy Hall. Su protagonista, Sydney Novak (Sophia Lillis), es una chica de diecisiete años con superpoderes que se enamora de su mejor amiga. En esa línea, ambos personajes son modelos que construyen un nuevo imaginario sobre la homosexualidad adolescente en la cultura popular. 


\section{DISERTACIONES}

ESTUDIOS

Grupos minoritarios y estigmatizados: diversidad funcional, religiosa, étnica, afectivo-sexual o de identidad de

género en la comunicación

ISSN: $1856-9536$

Doi: https://doi.org/10.12804/revistas.urosario.edu.co/disertaciones/a.10388

Volumen 15, Número 1 / Enero-junio 2022

Versión PDF para imprimir desde

http://revistas.urosario.edu.co/index.php/disertaciones

\section{La sociedad ante los contenidos audiovisuales: representaciones de la homosexualidad y consumo adolescente}

En la década de los noventa, el British Centre of Contemporary Cultural Studies puso en marcha una herramienta de análisis cultural que denominó The Circuit of Culture (Leve, 2012). Este "circuito de la cultura" es un modelo teórico y analítico para la investigación de la cultura popular, en concreto, del pensamiento académico y la producción artística (Velázquez, 2013), propuesto por Stuart Hall en el cual el producto cultural se concibe como un proceso de intercambio de significados con el que dar sentido al mundo (Leve, 2012). Según Hall, la representación consiste en dar forma física a una idea abstracta o en convertir el imaginario en algo concreto.

Las representaciones son signos que se utilizan para hacer referencia a un concepto que puede ser real o no, pero que facilita la comunicación entre dos seres humanos. Se pueden diferenciar dos maneras de representar: por un lado, la de las ideas mentales que el ser humano genera sobre objetos tangibles, y por otro, la de las relaciones, sentimientos y demás elementos abstractos; ambas formas de representación aparecen en un guion. En resumen, la representación sirve para precisar qué es un determinado concepto, y con ello, también se define aquello que no lo es. En definitiva, las representaciones afectan a aquellos significados del mundo real que se adoptan como válidos, son parte de la producción y del consumo de la sociedad occidental contemporánea (Leve, 2012). Una sociedad que ha aumentado el consumo de ficción seriada y donde el público objetivo adolescente "otorga a la ficción la función de entretenimiento, pero también le atribuye la función informativa y diversas funciones sociales" (Fedele \& García-Muñoz, 2010, p. 11).

Para comprender mejor la sociedad del momento y sus representaciones, debe tenerse en cuenta que "la identidad sexual en las series de ficción televisiva no puede estar divorciada de la pertenencia social y racial" (Ramírez Alvarado \& Cobo Durán, 2013, p. 232). Sin embargo, la aparición de la homosexualidad en las series de ficción ha sido progresiva. La cultura ha marcado la representación de los iconos aceptados por la sociedad donde las representaciones de género se basaban en el patriarcado y la heterosexualidad como norma. Evidenciando así la existencia de un sistema de relaciones de poder "que dan soporte a las epistemologías hegemónicas y sus representaciones en términos de imaginario social” (Martínez Portugal, 2020, p. 334).

Ahora bien, los estudios de género comenzaron a consolidarse en la academia anglosajona en la década de los setenta, aunque a mediados del siglo xx ya se había introducido el concepto "género" como constructo (Lamas, 2000). Este concepto se implantó progresivamente como herramienta de las ciencias sociales tales como la psicología, la filosofía, la educación, la sociología, la antropología y la historia (Bogino Larrambebere, 2017). La socialización de los seres humanos está intervenida por unas diferencias artificiales estipuladas por una cultura y sociedad determinadas (Pacheco Mejía, 2007). Mientras que en términos biológicos se nace con un sexo (varón o hembra), cultural y socialmente se nos asigna la masculinidad (en el caso del varón) o la feminidad (en el caso de la hembra), para determinar una serie de comportamientos y cualidades que todo ser humano debe alcanzar (Loncar, 2016).

El origen de los estudios queer es difícil de definir claramente, ya que surge de múltiples contextos críticos y culturales, incluido el feminismo, la teoría postestructuralista, los movimientos de gays y lesbianas, el poscolonialismo o el activismo contra el SIDA. El activismo gay y lésbico despierta con fuerza con el 68, dicha militancia se 


\section{DISERTACIONES}

ESTUDIOS

Grupos minoritarios y estigmatizados: diversidad funcional, religiosa, étnica, afectivo-sexual o de identidad de

género en la comunicación

ISSN: $1856-9536$

Doi: https://doi.org/10.12804/revistas.urosario.edu.co/disertaciones/a.10388

Volumen 15, Número 1 / Enero-junio 2022

Versión PDF para imprimir desde

http://revistas.urosario.edu.co/index.php/disertaciones

reconoce fácilmente en el hecho de que el término "queer" se toma como una nueva y orgullosa señal de identidad (Turner, 2000). Como herramienta académica, la denominada queer theory es entendida como método para deconstruir las identidades estigmatizadas (Fonseca \& Quintero, 2009). Su origen puede encontrarse en los estudios de género y sexualidad que, a su vez, tuvieron su origen en la teoría feminista y los estudios sobre lesbianas y gays. Establecida en la década de 1990, comienza cuestionando ideas como las categorías de identidad definidas y finitas. Uno de los textos fundacionales de la teoría queer es El género en disputa: el feminismo y la subversión de la identidad (Gender Trouble: Feminism and the Subversion of Identity), publicado en 1990 por Judith Butler, y en el que indica que "no existe una secuencia lógica entre naturaleza-sexo y cultura-género" (Butler citada en García-Santesmases, 2014, p. 21). Por su parte, en 1991 Teresa de Lauretis en Queer Theory: Lesbian and Gay Sexualities acuña el término "teoría queer" señalando que entre sus objetivos está el rechazo a la heterosexualidad como referente de las orientaciones sexuales y el repensar todo sobre la sexualidad. Para los estudios queer, "el género produce subjetividades más allá de la hegemonía masculina heterosexual [...] el sexo no es un rasgo innato, sino una identidad en construcción" (Mérida Jiménez, 2002, p.15). Los estudios queer han calado en la cultura popular actual de forma que cada vez son más las ficciones emitidas por los medios que presentan gran variedad temática y distintos enfoques dentro de los estereotipos de género; aunque sigue existiendo "un marcado sesgo androcéntrico, e incluso machista en muchas ocasiones" (Pibernat, 2017, p. 541). No obstante, la visibilidad homosexual en la televisión es cada vez mayor y su evolución progresiva:

La visibilidad homosexual ha pasado, en el discurso televisivo, como lo hiciera en el cinematográfico, por diferentes fases de representación: Oculta, Marginalizadora, Reivindicativa (en sus versiones integradora y transgresora) e Integrada. En poco más de dos décadas ha conseguido instalarse como protagonista en las series de prime time en virtud de una estrategia narrativa de visibilización integradora que responde a tres parámetros: respeto inicial de los estereotipos dominantes, respeto de los tabúes en la representación sexo/ género y observación de las convenciones narrativas precedentes. (Alfeo \& González de Garay, 2010, p. 18)

En este sentido, y siguiendo a los autores, se podría decir que el panorama televisivo contemporáneo experimenta la fase integrada. De hecho, en una reciente publicación, la misma González de Garay junto a María Marcos Ramos señala que en la actualidad se da "una tendencia hacia la igualdad de género y una mayor representación de las personas de la comunidad lgtb en plataformas in streaming" (2021, p. 596). Sin embargo, estas autoras indican que continúan existiendo aspectos de subrepresentación, tales como la crianza o profesiones específicas que siguen siendo consideradas femeninas. Así mismo, comportamientos que alguna vez estuvieron asociados con los varones, como la violencia, han sido adquiridos progresivamente por personajes femeninos de forma que los estereotipos que representan a las mujeres y las tramas en las que estas están involucradas han cambiado (2021, p. 596).

En cuanto a la adolescencia, Unicef la define como una etapa de desarrollo fundamental subrayando la importancia de este periodo, cuyos derechos están recogidos en la Convención sobre los Derechos del Niño que tuvo lugar en 1989. ${ }^{1}$

1 "Los adolescentes tienen derechos - tal como estableció en 1989 la Convención sobre los Derechos del Niño-y entre ellos se encuentra el derecho a la información y a adquirir conocimientos; a acceder a servicios, como la educación, la salud, los entretenimientos y la justicia; a un entorno seguro y estimulante, y a disponer de oportunidades para participar y expresar sus opiniones” (Unicef, 2002, p. 2). 


\section{DISERTACIONES}

ESTUDIOS

Grupos minoritarios y estigmatizados: diversidad funcional, religiosa, étnica, afectivo-sexual o de identidad de

género en la comunicación

ISSN: $1856-9536$

Doi: https://doi.org/10.12804/revistas.urosario.edu.co/disertaciones/a.10388

Volumen 15, Número 1 / Enero-junio 2022

Versión PDF para imprimir desde

http://revistas.urosario.edu.co/index.php/disertaciones

Desde el punto de vista físico, los niños pasan, de un día para otro, de ser pequeñas criaturas a convertirse en muchachos de largas piernas y brazos. Maduran sexualmente. También desarrollan la capacidad de razonar con ideas más abstractas, de explorar los conceptos del bien y del mal, de desarrollar hipótesis y de meditar sobre el futuro. (Unicef, 2002, p. 2)

En la actualidad, ante un contexto de socialización complejo, diverso y altamente mediatizado, son los propios adolescentes quienes mejor pueden expresar qué significa "crecer como hombre o mujer en la era digital" (Pibernat, 2017, p. 539). No debe olvidarse que la recepción de las representaciones mediáticas exige que la audiencia interprete los contenidos en función de sus expectativas, conocimientos y capacidades (Fedele \& García-Muñoz, 2010). En la etapa adolescente como periodo previo a la edad adulta y abandono de la infancia, la búsqueda de referentes es crucial, de forma que la exposición a arquetipos diversos puede influir positivamente en el desarrollo individual del joven espectador o espectadora.

Este interés por el consumo de contenidos audiovisuales, en los que la audiencia busca verse representada, se refleja en la teoría del cultivo propuesta por Gerbner en 1980, la cual defendía que los espectadores buscan un reflejo del mundo real en los contenidos televisivos. Por su parte, la teoría de la identificación, acuñada por Feilitzen y Linne en 1975, consideraba que es un proceso psicológico en el que un espectador desea ser como el personaje al que admira. Al respecto, la inclusión de adolescentes en las series de ficción se descubre como clave para que busquen identificarse con dichos personajes si los perciben como exitosos, admirados o atractivos (Di Pietro, 2018). El periodo adolescente entre los 14 y los 17 es, sin embargo, bastante turbulento en relación a los procesos de identificación (Ellithorpe \& Bleakley, 2016), un proceso que a partir de los 19 años comienza a decaer (Kroger et al., 2010).

Los y las adolescentes no han dejado de ver televisión, sino que eligen el espacio digital, cuya principal característica es la falta de limitación en el acceso. Esto les permite ver el contenido de forma inmediata, en cualquier lugar y controlar el tiempo que destinan para tal fin. Por lo tanto, en la actualidad el público adolescente "no deja de ver la televisión, sino que se emplean otros dispositivos, siempre con una orientación clara hacia el entretenimiento" (García Jiménez et al., 2018, p. 38). Esto es, los jóvenes continúan consumiendo televisión, siendo la ficción el consumo primordial. El presente texto articula cómo las representaciones de adolescentes gays y lesbianas subvierten de algún modo el orden patriarcal al ofrecer a los jóvenes nuevos y positivos imaginarios, entre los que se encuentran los personajes a los que se dedica el presente análisis: Danny Dixon y Sydney Novak.

\section{Metodología}

La representación en los medios de comunicación de las diversas orientaciones sexuales facilita la comprensión de la sociedad actual. Las dos primeras décadas del nuevo milenio se han convertido en un periodo clave para la visibilización de representaciones gays y lesbianas en la ficción audiovisual. Con el objetivo principal de revisar si la diferencia de género afecta también en las representaciones de la homosexualidad adolescente, se han elegido dos personajes, un varón y una mujer, que además permiten de manera secundaria abordar cómo se mueven estos personajes en la acción y determinar cómo se han construido. 


\section{DISERTACIONES}

Grupos minoritarios y estigmatizados: diversidad funcional, religiosa, étnica, afectivo-sexual o de identidad de género en la comunicación

Como se mencionó, se seleccionaron las series A Million Little Things (ABC, 2018) y Esta mierda me supera (Netflix, 2020) como objeto de análisis por el protagonismo de sus personajes adolescentes homosexuales y por ser producciones muy recientes, estrenadas en el 2018 y el 2020. Si bien ambas series de ficción estadounidenses no han alcanzado altas cotas de popularidad, su presencia internacional les ha permitido llegar a diversas audiencias y públicos como el español. Así bien, por un lado, se analiza a Sydney Novak (Esta mierda me supera), una joven de diecisiete años que descubre que está enamorada de su mejor amiga; y por otro, a Danny Dixon (A Million Little Things), un adolescente de doce años, que le cuenta a su madre que es gay. En este sentido, la homosexualidad de ambos personajes se manifiesta de forma explícita, siendo una trama principal.

Para llevar a cabo esta investigación de carácter cualitativo, siguiendo los objetivos planteados, se realizó un análisis narrativo desde el punto de vista psicológico de los dos personajes a partir de la propuesta metodológica sobre análisis de personajes de Carlos Grossocordón (2019). Dado que el objetivo era observar la evolución del personaje de ficción y la representación de la homosexualidad, se ha seleccionado la ficha técnica de análisis centrada en la dimensión psicológica. Como señala este autor, la dimensión psicológica está constituida por la personalidad y los aspectos psicológicos que constituyen el interior de un personaje (ambiciones; objetivos primarios/secundarios; frustraciones; temperamento; actitud ante la vida; carácter; tendencias; arco de transformación; tipo de conflicto; virtudes; defectos; carisma; vida afectiva sexual-moral; autoestima y complejos; supersticiones; imaginación e inteligencia) (Grossocordón, 2019). Asimismo, siguiendo a Grossocordón se han elaborado previamente las siguientes tablas, que recogen la información básica del personaje y contienen sus datos más relevantes (nombre, apellidos, época, clase, tipología y figura) (Grossocordón, 2019).

Tabla 1. Análisis de la información básica de Danny Dixon (A Million Little Things)

\begin{tabular}{|c|c|}
\hline Nombre & Danny \\
\hline Apellido & Dixon \\
\hline Época & Actual (2018) \\
\hline Clase & Personaje secundario \\
\hline Tipología & Arquetipo narrativo \\
\hline Figura & Auxiliar/ayudante de su madre (Delilah) \\
\hline
\end{tabular}

Fuente: elaboración propia a partir de la propuesta de Grossocordón (2019). 


\section{DISERTACIONES}

Grupos minoritarios y estigmatizados: diversidad funcional, religiosa, étnica, afectivo-sexual o de identidad de género en la comunicación

ISSN: $1856-9536$

Doi: https://doi.org/10.12804/revistas.urosario.edu.co/disertaciones/a.10388

Volumen 15, Número 1 / Enero-junio 2022

Versión PDF para imprimir desde

http://revistas.urosario.edu.co/index.php/disertaciones

Tabla 2. Análisis de la información básica de Sydney Novak (Esta mierda me supera)

\begin{tabular}{|c|c|}
\hline Nombre & Sydney \\
\hline Apellido & Novak \\
\hline Época & Actual (2020) \\
\hline Clase & Personaje principal, protagonista \\
\hline Tipología & Arquetipo narrativo \\
\hline Figura & Heroína / antiheroína \\
\hline
\end{tabular}

Fuente: elaboración propia a partir de la propuesta de Grossocordón (2019).

La información recogida en las tablas sobre los personajes se elaboró de acuerdo con el visionado de las temporadas disponibles hasta el momento de la elaboración de esta investigación. En el caso de Esta mierda me supera, la temporada se compone de siete episodios; y en A Million Little Things, de tres temporadas que constan de 49 episodios.

\section{Resultados y discusión}

Como se observa a continuación en las tablas 3 y 4, el arco de transformación es moderado en ambos personajes y se basa en la aceptación, tanto individual como del entorno. Cabe señalar que, tanto Sydney como Danny tienen en común la pérdida de la figura paterna, un hecho que los condiciona a nivel narrativo y de representación, por ejemplo, en el nihilismo y apatía de Sydney. Los dos han perdido a sus respectivos padres hace poco tiempo de la misma forma traumática: el suicidio. Esta circunstancia también afecta a sus respectivos intereses amorosos, y a otros personajes relacionados como Delilah (Stephanie Szostak), la madre de Danny en A Million Little Things. Precisamente, mientras que Sydney es la heroína protagonista de su propia historia, Danny es, más bien, un personaje satélite que gira en torno a Delilah, quien tiene que lidiar con su viudedad, los asuntos que dejó su esposo sin resolver y el cuidado de sus dos hijos adolescentes.

Tabla 3. Análisis de la dimensión psicológica de Danny Dixon (A Million Little Things)

\begin{tabular}{ll}
\hline Arco de transformación & Moderado \\
\hline Ambiciones & No se muestran \\
\hline Objetivos primarios/secundarios & Ir al instituto, pasar tiempo con su familia y conquistar a Elliot \\
\hline Tipo de conflicto & Interno (tiene miedo de que su madre no acepte su homosexualidad) \\
\hline Frustraciones & No aparecen \\
\hline
\end{tabular}




\section{DISERTACIONES}

\section{ESTUDIOS}

Grupos minoritarios y estigmatizados: diversidad funcional, religiosa, étnica, afectivo-sexual o de identidad de género en la comunicación

ISSN: 1856-9536

Doi: https://doi.org/10.12804/revistas.urosario.edu.co/disertaciones/a.10388

Volumen 15, Número 1 / Enero-junio 2022

Versión PDF para imprimir desde

http://revistas.urosario.edu.co/index.php/disertaciones

\begin{tabular}{ll}
\hline Temperamento/actitud ante la vida & Positivo \\
\hline Carácter/tendencias & Tiene buen humor y es extrovertido \\
\hline Virtudes & Es tierno, familiar, con buen carácter y sensible \\
\hline Defectos & No aparecen \\
\hline Carisma & Bondad \\
\hline Vida afectiva & Enamorado de Elliot \\
\hline Autoestima/complejos & $\begin{array}{l}\text { No parece tener, aunque está preocupado por la reacción del entorno a su } \\
\text { homosexualidad }\end{array}$ \\
\hline Supersticiones & No aparecen \\
\hline Imaginación & No se muestra \\
\hline Inteligencia & Es un chico muy perspicaz \\
\hline
\end{tabular}

Fuente: elaboración propia a partir de la propuesta de Grossocordón (2019).

Tabla 4. Análisis de la dimensión psicológica de Sydney Novak (Esta mierda me supera)

\begin{tabular}{|c|c|}
\hline Arco de transformación & Moderado \\
\hline Ambiciones & Controlar sus impulsos \\
\hline Objetivos primarios/secundarios & Que nadie descubra sus superpoderes \\
\hline Tipo de conflicto & Interno \\
\hline Frustraciones & No haber podido evitar que su padre se suicidara \\
\hline Temperamento/actitud ante la vida & $\begin{array}{l}\text { Cínica, apática e introvertida, excepto con su hermano, con su amigo Stan } \\
\text { y con su amiga Dina }\end{array}$ \\
\hline Carácter/tendencias & Cuando contiene sus enfados es capaz de hacer que se muevan los objetos \\
\hline Virtudes & $\begin{array}{l}\text { Tierna y divertida cuando está con sus amigos, cuida de su hermano } \\
\text { pequeño y ayuda a su madre en las tareas del hogar }\end{array}$ \\
\hline Defectos & $\begin{array}{l}\text { Evita expresar sus sentimientos reaccionando de manera agresiva. } \\
\text { No controla sus impulsos }\end{array}$ \\
\hline Carisma & Con sus amigos y su hermano: carisma de focalización y de bondad \\
\hline Vida afectiva & Enamorada de Dina y tiene un affair con Stan \\
\hline Autoestima/complejos & Autoestima baja, no se siente guapa ni inteligente \\
\hline
\end{tabular}




\section{DISERTACIONES}

ESTUDIOS

Grupos minoritarios y estigmatizados: diversidad funcional, religiosa, étnica, afectivo-sexual o de identidad de

\begin{tabular}{ll}
\hline Supersticiones & No aparecen \\
\hline Imaginación & No la muestra \\
\hline Inteligencia & Es buena estudiante \\
\hline
\end{tabular}

Fuente: elaboración propia a partir de la propuesta de Grossocordón (2019).

En ambas series, la homosexualidad adolescente se vincula a la importancia del papel maternal, más aún cuando las respectivas familias se convierten en monoparentales tras la pérdida de la figura paterna. Sin embargo, mientras que Sydney rechaza a su madre, Maggie (Kathleen Rose Perkins), Danny está muy unido a ella. De hecho, tras la muerte del padre, en pleno luto, Danny decide confesarle que su "novia Ella" es, en realidad, un diminutivo de "Elliot" (Bodhi Sabongui), su novio. Se aprecia, por tanto, la complicada relación madre-hija adolescente en Esta mierda me supera. Si bien Maggie es descrita como una madre sacrificada y ausente, ya que trabaja doce horas al día para poder pagar las facturas, su esfuerzo se convierte en una falta de comunicación con su hija. Ninguna es capaz de mantener una conversación sin gritar o lanzarse reproches. Todo lo contrario a lo que sucede en $A$ Million Little Things, donde Delilah no solamente acepta que su hijo es gay, sino que le anima a comenzar una relación con Elliot llegando a comentarles a los dos que "es precioso verlos juntos" (A Million Little Things, 2018, 15, min. 38). Danny también recibe apoyo por parte de otros adultos que refuerzan su autoestima, como Gary (James Roday Rodríguez), un amigo de su padre, que le exime de culpas explicándole que su homosexualidad no tuvo nada que ver en el suicidio de este, es más, "que su reacción habría sido de quererle aún más" (A Million Little Things, 2019, 3, min. 22). Así, además de la figura materna como central, parece que el padre debe estar ausente del relato, como si la figura patriarcal fuera un obstáculo o incompatible con el devenir homosexual del personaje. Esto por supuesto se puede vincular con las representaciones audiovisuales estereotípicas que de forma tradicional se han hecho de la homosexualidad, como las ligadas al trauma o a la tragedia. Según Ellithorpe y Bleakley (2016), es más común que los y las adolescentes busquen contenidos audiovisuales en los que se exhiban las diferentes orientaciones sexuales. De forma que los jóvenes buscan mensajes mediáticos con los cuales identificarse y desarrollar su identidad.

En este sentido, se puede observar cómo la protagonista lesbiana y adolescente angustiada, pero heroína en tanto posee superpoderes, tiene cabida en la plataforma Netflix, proyectada a alcanzar una audiencia juvenil; mientras que la representación de un adolescente gay encuentra su ubicación en productos audiovisuales emitidos en canal abierto y destinados al público adulto.

Por otro lado, debe tenerse en cuenta el consumo que la audiencia adolescente hace de los medios para comprender el análisis planteado. Tal y como indican Ferrán Casas et al. (2007, p. 329), "existen interacciones entre el género, la generación a la que se pertenece, el uso de las tecnologías y su consumo o las denominadas 'culturas de género'”. Al mismo tiempo, en el caso español los adultos consumen más contenido emitido en televisión y los adolescentes optan más por los VOD (Malo Cerrato et al., 2012). Además, al ser las chicas las grandes consumidoras de redes sociales frente a los chicos, que eligen, en su mayoría, el consumo de videojuegos, nos da una pista de que ellas leen los posts en las redes sociales sobre los contenidos de los canales VOD, que las vincula al 


\section{DISERTACIONES}

ESTUDIOS

Grupos minoritarios y estigmatizados: diversidad funcional, religiosa, étnica, afectivo-sexual o de identidad de

género en la comunicación

ISSN: $1856-9536$

Doi: https://doi.org/10.12804/revistas.urosario.edu.co/disertaciones/a.10388

Volumen 15, Número 1 / Enero-junio 2022

Versión PDF para imprimir desde

http://revistas.urosario.edu.co/index.php/disertaciones

consumo de este tipo de productos audiovisuales (Matrix, 2014). Esta circunstancia permite comprender cómo la protagonista adolescente lesbiana tiene cabida en Netflix, mientras que el personaje secundario gay es incluido en una serie de un canal en abierto.

La elección de la $A B C$ para incluir un personaje varón homosexual y adolescente en horario de prime time frente a la lesbiana protagonista en Netflix, refuerza la hipótesis de Ramírez Alvarado y Cobo Durán (2013) sobre que representar homosexuales en las series de televisión emitidas en horario de máxima audiencia beneficia a gays y lesbianas en su percepción social: "La mera representación de personajes gay masculinos en las series televisivas emitidas en prime time ha repercutido en las actitudes positivas hacia gays y lesbianas cambiando las nociones heteronormativas de la masculinidad y los modelos hegemónicos de las relaciones sociales" (2013, p. 230). Tras el análisis planteado, puede indicarse que esta es la estrategia seguida también en A Million Little Things, con el objetivo de "normalizar" entre una audiencia amplia y diversa la homosexualidad a través del personaje de Danny.

Esta exploración de la visibilidad se observa en las tramas de la serie en las que Danny y Elliot desarrollan su relación sentimental, por ejemplo, cuando tienen una cita para ver una película juntos (A Million Little Things, 2018, 15, min. 17). Además, el hecho de que se trate de ABC (American Broadcasting Company) - una de las cuatro cadenas de televisión más grandes de los Estados Unidos, creada en 1943 y en la actualidad propiedad de The Walt Disney Company-, puede entenderse como una estrategia para llegar a un mayor número de público, planteando así una mayor aceptación social de la homosexualidad por parte de la audiencia heterosexual. En el caso de Esta mierda me supera, al encontrarse en un canal VOD, los espectadores solamente pueden acceder pagando, aunque sin franja horaria limitada. No obstante, Netflix es reconocida como una plataforma que ha apostado porque sus contenidos siempre integren personajes LGBTQ+, no solo de manera secundaria, sino que sean los protagonistas de las historias.

\section{Conclusiones}

El análisis de los dos personajes adolescentes propuestos, Danny Dixon de A Million Little Things (ABC, 2018) y Sydney Novak de Esta mierda me supera (Netflix, 2020) permite concluir que en la actualidad hay cierto interés por visibilizar la homosexualidad durante la etapa adolescente abordada desde la cotidianeidad, esto es, sin que los personajes lo experimenten con dramatismo o histrionismo, como sucedía tradicionalmente. La homosexualidad como tropo se inserta no solo en las ficciones dirigidas a un target o público juvenil, sino también en los contenidos audiovisuales destinados a los adultos, ofreciendo una mayor comprensión a quienes lidian con los y las adolescentes, es decir, familiares y amistades que participan en su desarrollo personal y psicológico.

En el caso de Danny Dixon, su retrato ofrece una imagen del gay adolescente que, a pesar de su trauma familiar, posee madurez a la hora de relacionarse con los demás, y mantiene una relación sana con su madre. Su "salida del armario" no fue traumática, todo lo contrario, fue recibida con orgullo por su madre, del mismo modo que con Gary, un amigo de su padre. En cuanto a Sydney Novak, su orientación sexual no es tomada como un hecho clave para la conformación de su identidad. Sydney tiene que lidiar con otros problemas que considera mayores, como el cuidado de su hermano pequeño, la culpabilidad por la muerte de su padre o qué hacer con sus superpoderes. Si bien es cierto que su imagen resulta más problemática que la de Danny, debe recordarse que 


\section{DISERTACIONES}

ESTUDIOS

Grupos minoritarios y estigmatizados: diversidad funcional, religiosa, étnica, afectivo-sexual o de identidad de

se trata de la protagonista del relato, y que, por tanto, tiene más matices. Debe señalarse además el convencionalismo estereotipado en el que los personajes femeninos "empoderados" -en tanto que Sydney tiene superpoderes-, deban poseer a cambio ciertas taras, particularmente, como en su caso una mala relación maternofilial y el carácter introvertido entre otras, en definitiva, cierta angustia adolescente femenina. A pesar de ello, se trata de una protagonista interesante como arquetipo: Sydney toma sus propias decisiones, no se somete a ningún poder masculino, al tiempo que no es sexualizada ni tampoco masculinizada.

A pesar de que tanto Danny como Sydney coinciden en el sufrimiento inicial de un hecho traumático -el suicidio del padre-, que pueden situar al personaje en el dramatismo, ambos ofrecen nuevos y positivos retratos de la homosexualidad, en este caso a través de la adolescencia. Los dos personajes - teniendo en cuenta la distancia entre ambas series de ficción- articulan una presentación de los personajes homosexuales muy alejada de los estereotipos negativos que tradicionalmente han presentado la televisión y el cine. De esta manera, que los personajes de lesbianas y gays incrementen su protagonismo permite abrir y normalizar diversos estilos de vida y de orientaciones sexuales al gran público, logrando no solo una mayor visibilidad sino también una mayor aceptación por parte de la audiencia heterosexual. En definitiva, la aparición de personajes lesbianas y gays adolescentes en las series de televisión es una apuesta positiva a nivel estratégico empresarial (las cadenas se hacen eco de las tendencias lgtb-friendly), pero más aún como herramienta de socialización. Mostrar imágenes en las que los y las adolescentes puedan sentirse representados, así como poner en situación a sus tutores y progenitores para que aprehendan la orientación sexual de sus hijos o hijas refuerza la aceptación de la propia diversidad sexual. Asimismo, la integración de las representaciones de adolescentes homosexuales facilita la aceptación de que esta vía es posible por parte de los adultos desde una temprana edad, o más bien, a partir de que la personalidad comienza a manifestarse en ese periodo de vida tan complicado y maravilloso que se encuentra entre la etapa infantil y la edad adulta.

\section{Referencias}

1. Alfeo Álvarez, J. C., \& González de Garay, B. (2010). Negociación de la visibilidad homosexual en la ficción televisiva española. En La Construcción de Género en la Ficción televisiva (p. 11). Servei de Publicacions.

2. Bogino Larrambebere, M. R. (2017). Relecturas de género: concepto normativo y categoría crítica. La Ventana. Revista de Estudios de Género, 5(45), 158-185. http://www.scielo.org.mx/scielo. php?pid=S1405-94362017000100158\&script=sci_abstract\&tlng=pt

3. Butler, J. (1990). El género en disputa: El feminismo y la subversión de la identidad. Paidós.

4. Casas Aznar, F., Figuer, C., González Carrasco, M., \& Malo Cerrato, S. (2007). Los medios audiovisuales entre los progenitores y los hijos e hijas. Culture and Education, Cultura y Educación, 19(3), 311-330. https://doi. org/10.1174/113564007782194499

5. De Lauretis, T. (1991). Queer theory: Lesbian and gay sexualities. A Journal of Feminist Cultural Studies, 3(2).

6. Di Pietro, G. (2018). Do media play a role in promoting vocational education and training? The case of MasterChef. Policy Studies, 39(1), 37-53. https://doi.org/10.1080/01442872.2017.1410879

7. Durand, G. (2004). Las estructuras antropológicas del imaginario. Fondo de Cultura Económica. 


\section{DISERTACIONES}

ESTUDIOS

Grupos minoritarios y estigmatizados: diversidad funcional, religiosa, étnica, afectivo-sexual o de identidad de

género en la comunicación

ISSN: $1856-9536$

Doi: https://doi.org/10.12804/revistas.urosario.edu.co/disertaciones/a.10388

Volumen 15, Número 1 / Enero-junio 2022

Versión PDF para imprimir desde

http://revistas.urosario.edu.co/index.php/disertaciones

8. Ellithorpe, M., \& Bleakley, A. (2016). Wanting to see people like me? Racial and gender diversity in popular adolescent television. Journal of Youth and Adolescence, 45(7), 1426-1437. https://doi.org/10.1007/ s10964-016-0415-4

9. Fedele, M., \& García-Muñoz N. (2010). El consumo adolescente de la ficción seriada. Vivat Academia, (111), 47-64. https://doi.org/10.15178/va.2010.111.47-64

10. Fonseca, C., \& Quintero, M. L. (2009). La Teoría Queer: la de-construcción de las sexualidades periféricas. Sociológica (México), 24(69), 43-60. http://www.scielo.org.mx/scielo.php?script=sci_arttext\&pid= S0187-01732009000100003

11. García Jiménez, A., Tur-Viñez, V., \& Pastor Ruiz, Y. (2018). Consumo mediático de adolescentes y jóvenes. Noticias, contenidos audiovisuales y medición de audiencias. Icono 14, 16(1), 22-46. http://dx.doi. org/10.7195/ri14.v16i1.1101

12. García-Santesmases Fernández, A. (2014). Dilemas feministas y reflexiones encarnadas: el estudio de la identidad de género en personas con diversidad funcional física. Athenea Digital. Revista de Pensamiento e Investigación Social, 14(4), 19-47. https://raco.cat/index.php/Athenea/article/view/292028

13. Gerbner, G. (2002). Against the mainstream: The selected works of george gerbner. Media and Culture. Peter Lang.

14. González de Garay, B., \& Marcos Ramos, M. (2021). gender representation in subscription video-on-demand spanish tv Series. International Journal of Communication, 15, 581-604. https://acortar.link/bvg206

15. Grossocordón, C. (2019). Propuesta metodológica sobre análisis de personajes en el relato cinematográfico. Comunicación y Métodos, 1(1), 9-28. https://doi.org/10.35951/v1i1.18

16. Kroger, J., Martinussen M., \& James, E.M. (2010). Identity status change during adolescence and young adulthood: A meta-analysis. Journal of Adolescence, 33(5), 683-698. https://doi.org/10.1016/j. adolescence.2009.11.002

17. Lamas, M. (2000). El género. La construcción cultural de la diferencia sexual. Miguel Ángel Porrúa.

18. Leve, A. M. (2012). The circuit of culture as a generative tool of contemporary analysis: examining the construction of an education community. apere Conference, Sidney.

19. Loncar, M. (2016). Constructing Masculinity through Images. World Academy of Science, Engineering and Technology International Journal of Social, Behavioral, Educational, Economic, Business and Industrial Engineering, 10(10), 3123-3126. https://n9.cl/vecbv

20. Malo Cerrato, S., Navarro Pou, D., \& Casas Aznar, F. (2012). El uso de los medios audiovisuales en la adolescencia y su relación con el bienestar subjetivo: análisis cualitativo desde la perspectiva intergeneracional y de género. Athenea Digital, Revista de Pensamiento e Investigación Social, 12(3), 27-49. https://www. redalyc.org/pdf/537/53724611003.pdf

21. Martínez Portugal, T. (2020). Haciendo frente a las epistemologías heteropatriarcales: elementos teórico-metodológicos para un análisis feminista de la violencia contra las mujeres. Investigaciones Feministas, 11(2), 333-342.

22. Matrix, S. (2014). The Netflix effect: Teens, Binge watching, and on-demand digital media trends. Jeunesse: Young People, Texts, Cultures, 6(1), 119-138. https://doi.org/10.1353/jeu.2014.0002

23. Mérida Jiménez, R. M. (2002). Sexualidades transgresoras. Una antología de estudios queer. Icaria. 


\section{DISERTACIONES}

Grupos minoritarios y estigmatizados: diversidad funcional, religiosa, étnica, afectivo-sexual o de identidad de

24. Pacheco Mejía, E. E. (2007). El género en las cintas de Disney. Temas Antropológicos: Revista Científica de Investigaciones Regionales, 29(1-2), 87-106.

25. Pibernat Vila, M. (2017). ¿Nuevas socializaciones, viejas cuestiones? Adolescencia y género en la era audiovisual. Investigaciones Feministas: Papeles de Estudios de Mujeres, Feministas y de Género, 8(2), 529-544.

26. Ramírez Alvarado, M. M., \& Cobo Durán, S. (2013). La ficción gay-friendly en las series de televisión españolas. Comunicación y Sociedad, (19), 213-235. http://www.scielo.org.mx/scielo. php?script=sci_arttext\&pid=S0188-252X2013000100009

27. Unicef. (2002). Adolescencia: una etapa fundamental. Fondo de las Naciones Unidas para la Infancia. https://ibdigital.uib.es/greenstone/sites/localsite/collect/portal_social/index/assoc/unicef00/02.dir/unicef0002.pdf

28. Veissière, S.P.L. (2018). Toxic masculinity in the age of \#MeToo: Ritual, morality and gender archetypes across cultures. Society and Business Review, 13(3), 274-286. https://doi.org/10.1108/SBR-07-2018-0070

29. Velázquez, R. (2013). La tía Tula de Miguel Picazo a la luz del circuito de la cultura. Tonos Digital: Revista Electrónica de Estudios Filológicos, (24), 1-25. http://www.tonosdigital.es/ojs/index.php/tonos/article/ viewArticle/917

30. Von Feilitzen, C. (2008). Children and media literacy: Critique, practice, democracy. Doxa Comunicación: Revista Interdisciplinar de Estudios de Comunicación y Ciencias Sociales, (6), 317-332. http://agora.edu.es/ servlet/articulo?codigo $=2653854$ 